\title{
FINITE CORRESPONDENCE OF SPECTRA IN NOETHERIAN RING EXTENSIONS
}

\author{
EDWARD S. LETZTER
}

\author{
(Communicated by Maurice Auslander)
}

\begin{abstract}
Let $R \hookrightarrow S$ be an embedding of associative noetherian rings such that $S$ is finitely generated as a right $R$-module. There is a correspondence from the prime spectrum of $S$ to the prime spectrum of $R$ obtained by associating to a given prime ideal $P$ of $S$ the prime ideals of $R$ minimal over $P \cap R$. The prime and primitive ideal theories for several specific noncommutative noetherian rings, including group algebras, PI algebras, and enveloping algebras, depend on understanding instances of this correspondence. We prove that the correspondence has finite fibers for a class of noetherian ring extensions that unites these examples.
\end{abstract}

\section{INTRODUCTION}

Let $R$ and $S$ be noetherian rings; suppose that $S$ contains $R$ as a subring; and suppose that $S$ is finitely generated as a right $R$-module. Recall that if $Q$ is a prime ideal of $R$ and $P$ is a prime ideal of $S$, then $P$ lies over $Q$ provided $Q$ is minimal over $P \cap R$. Also recall, for a prime ideal $P$ of $S$ lying over a prime ideal $Q$ of $R$, that $P$ is left primitive if and only if $Q$ is left primitive when $S$ is finitely generated on both sides as an $R$-module [14]. The primary goal of this paper is to prove

Theorem A. If $Q$ is a prime ideal of $R$ and $n$ is a positive integer, then there exist at most finitely many prime ideals $P$ of $S$ lying over $Q$ such that the Goldie rank of $S / P$ is equal to $n$.

We are consequently able to show that only finitely many prime ideals of $S$ can lie over a given prime ideal $Q$ of $R$ given an additional technical hypothesis on $Q$. Included in the class of rings whose prime ideals satisfy this hypothesis are complex group algebras over polycyclic-by-finite groups, noetherian PI algebras, and enveloping algebras of finite-dimensional complex solvable or semisimple Lie algebras. Prime ideal structure in various ring extensions of these types has been extensively studied; examples will be presented in $\S 2$.

The graph of links of a noetherian ring is a certain directed graph structure imposed on the prime spectrum, and cliques are connected components. (The

Received by the editors July 17, 1990 and, in revised form, April 8, 1991.

1991 Mathematics Subject Classification. Primary 16D30, 16P40, 16 S99.

Key words and phrases. Prime ideal, primitive ideal, Noetherian ring, ring extension.

A part of this research was supported by an NSF Postdoctoral Research Fellowship. 
reader is referred to $[7,10,21]$ for the basic terminology of noetherian ring theory.)

Corollary B. Let $Q$ be a prime ideal of $R$, and suppose there exists a positive integer $m$ such that the Goldie rank of $R / I$ is less than $m$ for each prime ideal $I$ in the clique of $Q$. Then there exist at most finitely many prime ideals of $S$ lying over $Q$.

Our approach to the above results leads us to observe that under certain additional restrictions every left primitive ideal of $S$ can be obtained from a left primitive ideal of $R$ in the following way: Suppose that $R \hookrightarrow S$ is an embedding of algebras of finite Gelfand-Kirillov dimension (GK-dimension) over a field, and suppose that $S$ is finitely generated as both a left and right $R$-module. (See [12] or [21] for information about GK-dimension.) Let $Q$ be a left primitive ideal of $R$, and let $J_{Q}$ be the annihilator in $S$ of the left $S$ module $S_{R} \otimes \operatorname{Fract}(R / Q)$. Let $X_{Q}$ be the possibly empty but at most finite set of prime ideals of $S$ that are minimal over $J_{Q}$. Denote the set of left primitive ideals of a ring $A$ by $\operatorname{prim} A$. We see in (4.2) that

$$
\operatorname{prim} S=\bigcup_{Q \in \operatorname{prim} R} X_{Q}
$$

Applications of this fact will appear in $[16,17]$.

Our methods throughout are based on techniques and results developed to study additivity principles $[2,3,11,26]$, localization theory $[10,24]$, and representation theory $[5,10,13]$ for noncommutative noetherian rings. This paper is a continuation of our previous work in [14, 15], and our point of view is in part analogous to the approach that was taken in [23].

This paper is an improved version of a preprint that was circulated in the spring of 1989. In the earlier version Theorem A and Corollary B were proved for an extension of noetherian algebras of finite GK-dimension over a field. In addition, it was necessary to assume for the earlier proof that the extension ring was finitely generated on both the left and right as a module over the subring. The observations of $\S 4$ were more central to our original approach.

Notation and conventions. Throughout this note $R$ and $S$ denote noetherian rings such that $S$ contains $R$ as a subring and such that $S$ is finitely generated as a right $R$-module. The correspondence of spectra will always refer to the assignment to a prime ideal $P$ of $S$ the prime ideals of $R$ minimal over $P \cap R$. The classical quotient ring of a prime noetherian $\operatorname{ring} A$ will be denoted Fract $A$. The Goldie rank of a prime noetherian ring $A$ will be denoted by rank $A$. If $A$ is a $k$-algebra denote the GK-dimension over $k$ of an $A$-module $M$ by $\mathrm{d}(M)$. The set of prime ideals of a ring $A$ will be denoted $\operatorname{spec} A$.

\section{EXAMPLES}

2.1. Let $\mathbf{g}=\mathbf{g}_{0} \oplus \mathbf{g}_{1}$ be a complex finite-dimensional classical simple Lie superalgebra. Let $\mathbf{U}$ denote the enveloping algebra of the (reductive) Lie algebra $\mathbf{g}_{0}$, and let $\mathbf{V}$ denote the enveloping algebra of $\mathbf{g}$. Then $\mathbf{U}$ embeds in $\mathbf{V}$ and $\mathbf{V}$ is finitely generated as a right and left $\mathbf{U}$-module. (See [22] for details.) Since 
$\mathbf{g}_{0}$ is reductive, at most finitely many prime ideals of $\mathbf{U}$ can have a common intersection with the center of $\mathbf{U}$ [6]. Hence every clique of prime ideals of $\mathbf{U}$ is finite. So Corollary B shows that the correspondence of spectra is finite-tofinite if $R=\mathbf{U}$ and $S=\mathbf{V}$. (We do not know of a more direct proof of this fact.) It has been observed by Lenagan that the correspondence is surjective; see $[14,2.1]$.

2.2. Let $\mathbf{g}$ be a finite-dimensional complex semisimple Lie algebra; suppose that $R$ is a homomorphic image of the enveloping algebra $U$ of $\mathbf{g}$; and suppose that $S$ is an arbitrary noetherian ring containing $R$ finitely generated as a right $R$-module. Each clique of prime ideals of $R$ is finite, as noted for $\mathbf{U}$ in the previous paragraph. Hence it follows from Corollary B that the correspondence of spectra for $R \hookrightarrow S$ is finite-to-finite.

One well-known example of such an embedding arises as follows: Let $M$ be a faithful left $R$-module of finite length. There is a natural algebra embedding of $R$ into $\operatorname{Hom}_{\mathrm{C}}(M, M)$. (See, for example, [9, 6.2.b].) Next, let $\mathscr{L}(M, M)$ be the $R$-R-sub-bimodule of $\operatorname{Hom}_{\mathrm{C}}(M, M)$ comprised of all elements $\varphi$ such that $\{x \cdot \varphi-\varphi \cdot x: x \in \mathbf{g}\}$ is finite dimensional. Let $S=\mathscr{L}(M, M)$. The image of the above embedding of $R$ into $\operatorname{Hom}_{\mathrm{C}}(M, M)$ is contained within $S$. (See $[9,6.8]$.) Furthermore, $S$ is finitely generated on each side as an $R$-module and is of finite length as an $R$ - $R$-bimodule. (See [9, 6.10.b, 8.17.c].) Hence $S$ is a noetherian ring of finite length as an $S$-S-bimodule. Therefore, the prime spectrum of $S$ is finite. (This last assertion is undoubtedly known. As we do not know an explicit reference, we include a brief proof: Let $I$ denote the intersection of all nonminimal prime ideals of $S$. Since $S$ has finite length as an $S$-S-bimodule, $I$ is the intersection of finitely many nonminimal prime ideals. In particular, $I$ contains a finite product of nonminimal prime ideals. Consequently, $I$ cannot be contained within any minimal prime ideal of $S$. Now let $n$ denote the finite number of minimal prime ideals of $S$. Since $|\operatorname{spec} S| \leq|\operatorname{spec}(S / I)|+n$, the assertion follows by induction on the length of $S$ as an $S$-S-bimodule.) So it is essentially already known for this example that the correspondence is finite-to-finite. (The reader is referred, for example, to [9] for applications of the construction $\mathscr{L}(M, M)$.)

The algebras conjecturally introduced in $[25,1.26]$ provide examples that need not be bimodules of finite length.

2.3. Suppose that $R$ and $S$ satisfy a polynomial identity. It is shown in $[15,4.8]$ that the correspondence of spectra is surjective. There is a uniform bound for the Goldie ranks of prime factors of $R$ and $S$, so by Theorem A the correspondence of spectra is finite-to-finite. These results were proved in [1, 4.15] for $R$ and $S$ affine PI algebras finite over their centers. Included in the general example is the extension $A \hookrightarrow T(A)$, where $A$ is a prime noetherian PI ring and $T(A)$ is the trace ring of $A$. (See [20].) However, $A \hookrightarrow T(A)$ is a centralizing extension, so the conclusion of Corollary B is known for all prime ideals of $A$ by [19].

2.4. Let $K$ be a commutative noetherian ring, and let $G$ be a polycyclic-byfinite group. It is shown in [4] for each clique $\Omega$ of $K G$ that there exists a finite bound for the set $\{\operatorname{rank}(K G / I): I \in \Omega\}$. So suppose $R$ is a homomorphic 
image of $K G$. Then the hypothesis of Corollary B applies to all prime ideals of $R$. If $S$ is finitely generated as both a right and left $R$-module, then for each prime ideal $Q$ of $R$ there exists a prime ideal of $S$ lying over $Q$ by $[10$, A.4.6; 7, 12.16]. Included in this example is the extension $K G \hookrightarrow K H$, for $G$ a subgroup of $H$ of finite index. When $G$ is a normal subgroup of $H$ of finite index, the existence of finitely many prime ideals of $K G$ lying over each prime ideal of $K H$ follows from the detailed structure theory already available; see [18, 16.9ii]. (See also [18], especially $\S 16$, for more details and related examples.)

2.5. It may be the case for a given prime ideal $Q$ of $R$ that there exist no prime ideals of $S$ lying over $Q$. In [8] such an example $R \hookrightarrow S$ is constructed for $S$ finitely generated on both sides as an $R$-module.

\section{Proofs of Theorem A and Corollary B}

3.0. We begin with a well-known observation. (See, for example, [7, 7.21].) Let $P$ be a prime ideal of $S$, and let $Q$ be a prime ideal of $R$ minimal over $P \cap R$. Let $F=\operatorname{Fract}(S / P)$. Choose an $F$-R-bimodule composition series $0=F_{0} \subset \cdots \subset F_{q}$ of $F$, and let $Q_{i}=\operatorname{ann}\left(F_{i} / F_{i-1}\right)_{R}$ for each $1 \leq i \leq q$. Observe that $P \cap R \subseteq Q_{1}, \ldots, Q_{q}$, and recall that the right annihilator of a simple bimodule is prime. Therefore, since $Q_{1} \cdots Q_{q} \subseteq P \cap R$, and since $Q$ is a prime ideal of $R$ minimal over $P \cap R$, it follows that $Q=Q_{i}$ for some $1 \leq i \leq q$. In particular, there exists a simple $F$-R-bimodule subfactor of $F$ with right annihilator equal to $Q$.

Definition 3.1. (i) Let $P$ be a prime ideal of $S$ and let $F=\operatorname{Fract}(S / P)$. Let $\mathscr{V}_{P}$ denote the (finite) set of prime ideals of $R$ that are right annihilators in $R$ of simple $F$-R-bimodule factors of $F$.

(ii) Let $Q$ be a prime ideal of $R$, and let $A=\operatorname{Fract}(R / Q)$. Denote by $\mathscr{W}_{Q}$ the (possibly empty but at most finite) set of prime ideals of $S$ that are left annihilators in $S$ of simple $S$ - $A$-bimodule factors of $S_{R} \otimes A$.

Lemma 3.2. Let $P$ be a prime ideal of $S$. Then $Q \in \mathscr{V}_{P}$ if and only if $P \in \mathscr{W}_{Q}$.

Proof. Let $F=\operatorname{Fract}(S / P)$. Suppose first that $Q \in \mathscr{V}_{P}$. Choose a simple $F$-R-bimodule factor $G$ of $F$ such that $Q=$ ann $G_{R}$. From [7, 7.19(a)] we see that $G$ is torsionfree as a right $R / Q$-module. Let $H$ be the canonical $S$ - $R$-bimodule image of $S$ in $G$. Then $P=\operatorname{ann}_{S} H, Q=\operatorname{ann} H_{R}$, and $H$ is torsionfree as a right $R / Q$-module. Let $K=H_{R} \otimes A$. Then $K$ has finite length as a right $A$-module, $P=\operatorname{ann}_{S} K$, and $K$ is torsionfree as a left $S / P$-module. Since $P$ is the only left affiliated prime of $K$, it follows from $[7,7.20]$ that $P$ is the left annihilator in $S$ of a simple $S$ - $A$-bimodule factor of $K$. The "only if" assertion follows.

Now suppose that $P$ is the left annihilator of a simple $S$ - $A$-bimodule factor $L$ of $S_{R} \otimes A$. Let $M$ denote the canonical $S$-R-bimodule image in $L$ of $S$. It follows from [7,7.19(a)] that $L$ is torsionfree as a left $S / P$-module, and, therefore, $M$ is an $S$ - $R$-bimodule factor of $S / P$ that is torsionfree as a left $S / P$-module. Observe that $M$ is torsionfree as a right $R / Q$-module. So $Q$ is the only right affiliated prime of $F_{S} \otimes M_{R}$, an $F$-R-bimodule factor of 
$F$. It then follows from [7, 7.20] that $Q$ is the right annihilator of a simple $F$ - $R$-bimodule factor of $F$. The lemma follows.

Definition 3.3. Let $Q$ be a prime ideal of $R$. Denote by $\Omega(Q, m, n)$ the set of prime ideals $Q^{\prime}$ of $R$ satisfying the following property: There exists a set $\left\{Q=Q_{1}, \ldots, Q_{t}=Q^{\prime}\right\}$ of prime ideals of $R$ such that (i) $1 \leq t \leq m$, (ii) $\operatorname{rank}\left(R / Q_{i}\right) \leq n$ for all $1 \leq i \leq t$, and (iii) $\left\{Q_{1}, \ldots, Q_{t}\right\}$ is a connected subset of the graph of links of $R$. Let $\Omega(Q)=\bigcup_{n=1}^{\infty} \Omega(Q, n, n)$, the clique of $Q$.

Lemma 3.4. Let $Q$ be a prime ideal of $R$, let $n$ be a positive integer, let $P$ be a prime ideal of $S$ such that $\operatorname{rank}(S / P) \leq n$, and assume that $P$ lies over $Q$. Then $\mathscr{V}_{P} \cap \Omega(Q, n, n)=\varnothing$.

Proof. Let $F=\operatorname{Fract}(S / P)$. We first construct a suitable factor of $F$. From (3.0) it follows that $Q$ is the right annihilator in $R$ of a simple $F$-R-bimodule subfactor of $F$. Therefore, there exists an $F$-R-bimodule factor ${ }_{F} N_{R}$ of $F$ such that $Q$ is the right annihilator in $R$ of a simple $F$-R-sub-bimodule $U$ of $N$. Choose an $F$-R-sub-bimodule $U^{\prime}$ of $N$ maximal such that $U^{\prime} \cap U=0$. Let $M=N / U^{\prime}$; note that $U$ embeds essentially in $M$ as an $F$-R-sub-bimodule; and identify $U$ with its image. We deduce as follows that $Q$ is the only annihilator prime of $M_{R}$ : Let $Q^{\prime}$ be another annihilator prime of $M_{R}$, and let $V=\operatorname{ann}_{M} Q^{\prime}$. The simplicity and essentiality of the $F$ - $R$-sub-bimodule $U$ guarantee that $U \subseteq V$. Hence $Q^{\prime} \subseteq Q$. But $Q^{\prime} \supseteq P \cap R$, and $Q$ is minimal over $P \cap R$. So $Q=Q^{\prime}$, and it must be the case that $Q$ is the only annihilator prime of $M_{R}$. Moreover, letting $L$ denote the canonical $S$-R-bimodule image in $M$ of $S$, we see that $Q$ is the only annihilator prime of $L_{R}$.

Now choose a right affiliated series $0=M_{0} \subset \cdots \subset M_{t}=M$ of ${ }_{F} M_{R}$. Let $Q=\operatorname{ann}\left(M_{i} / M_{i-1}\right)_{R}$ for $1 \leq i \leq t$. We claim that $\left\{Q_{1}, \ldots, Q_{t}\right\} \subseteq$ $\Omega(Q, n, n)$ : First note that $t \leq n$. Next note that $0=M_{0} \cap L \subset \cdots \subset$ $M_{t} \cap L=L$ is a right affiliated series of the noetherian (on each side) bimodule ${ }_{S} L_{R}$. In the above paragraph we saw that $Q$ is the unique annihilator prime of $L_{R}$. It thus follows from an iterated application of [13,1.2], or from the proof of $[10,8.3 .1]$, that the set $\left\{Q_{1}, \ldots, Q_{t}\right\}$ is a connected subset of the graph of links of $R$. Finally, it follows from [7, 7.22] for $1 \leq i \leq t$ that $\operatorname{rank}\left(R / Q_{i}\right) \leq n$, since $M$ is a left $F$-module factor of $F$. The claim follows.

Note as a consequence of $[7,7.20]$ and the last paragraph that there exists a prime ideal $Q^{\prime}$ in $\Omega(Q, n, n)$ that is the right annihilator in $R$ of a simple $F$-R-bimodule factor of $M$. The lemma now follows since $M$ is an $F-R$ bimodule of $F$.

Remark 3.5. A theorem of Stafford [24, 3.10] (or see [7, 14.21]) states that $\Omega(Q, m, n)$ is finite for each prime ideal $Q$ of $R$ and each pair of positive integers $m, n$.

3.6. Proof of Theorem A. Let $Q$ be a prime ideal of $R$, let $n$ be a positive integer, and let $\mathscr{P}$ denote the set of prime ideals $P$ of $S$ lying over $Q$ such that $\operatorname{rank}(S / P)=n$. From (3.2) and (3.4) it follows that $\mathscr{P} \subseteq \bigcup_{I \in \Omega(Q, n, n)} \mathscr{W}_{I}$. Hence from (3.5) it follows that $\mathscr{P}$ is finite. 
Lemma 3.7. Suppose that $S=s_{1} \cdot R+\cdots+s_{t} \cdot R$, for elements $s_{1}, \ldots, s_{t}$ of $S$. Let $P$ be a prime ideal of $S$ and let $Q \in \mathscr{V}_{P}$. Then $\operatorname{rank}(S / P) \leq t \cdot \operatorname{rank}(R / Q)$.

Proof. Let $F=\operatorname{Fract}(S / P)$, and let $A=\operatorname{Fract}(R / Q)$. Choose a simple $F-R$ bimodule factor $N$ of $F$ such that ann $N_{R}=Q$, and let $M$ be the canonical $S$-R-bimodule image in $N$ of $S$. From [7, 7.19(a)] it follows that $M$ is torsionfree as a right $R / Q$-module. Let $L=M \otimes_{R} A$. Then $L$ is a torsionfree left $S / P$-module and has length no greater than $t \cdot \operatorname{rank}(A)$ as a right $A$-module. The lemma now follows from [7, 7.22].

3.8. Proof of Corollary B. Let $P$ be a prime ideal of $S$ lying over $Q$, and suppose that $S$ is generated as a right $R$-module by $t$ elements. By (3.4) there exists an element $I \in \mathscr{V}_{P}$ such that $I \in \Omega(Q)$. Therefore $\operatorname{rank}(S / P) \leq t m$ by (3.7). Consequently, there is a finite upper bound for the set of positive integers $\{\operatorname{rank}(S / J): J$ lies over $Q\}$, unless this set is empty. The desired conclusion now follows from Theorem $A$.

\section{INDUCED MODULES}

In this section assume that $S$ is finitely generated as both a right and left $R$-module, that $R$ and $S$ are algebras of finite $G K$-dimension over a field $k$, and that the inclusion of $R$ in $S$ is a $k$-algebra embedding. We have concentrated so far on the correspondence assigning to each prime ideal $P$ of $S$ those prime ideals of $R$ minimal over $P \cap R$. We now observe that a more explicit realization of the prime and primitive spectra of $S$ can be extracted from $R$ under the additional hypotheses.

4.1. For each prime ideal $Q$ of $R$, let $J_{Q}$ denote the annihilator in $S$ of the left $S$-module $S_{R} \otimes \operatorname{Fract}(R / Q)$. (Observe that if $S$ is free as a right $R$ module then $J_{Q}$ is the left annihilator in $S$ of $S / S Q$.) Let $X_{Q}$ denote the (possibly empty but at most finite) set of prime ideals of $S$ minimal over $J_{Q}$, and let $T_{Q}$ denote the kernel of the canonical $S$ - $R$-bimodule homomorphism $S \rightarrow S_{R} \otimes \operatorname{Fract}(R / Q)$. Note that $S / T_{Q}$ is an $S / J_{Q}-R / Q$-bimodule that is faithful and finitely generated on each side. Recall that an annihilator prime of a left $R$-module $M$ is a prime ideal $Q$ of $R$ such that $Q$ is equal to the annihilator of some left $R$-submodule of $M$.

Proposition 4.2. (i) $\operatorname{spec} S=\bigcup_{Q \in \operatorname{spec} R} X_{Q}$.

(ii) $\operatorname{prim} S=\bigcup_{Q \in \operatorname{Prim} R} X_{Q}$.

(iii) Let $P$ be a prime ideal of $S$, and let $Q$ be an annihilator prime of ${ }_{R}(S / P)$. Then $P \in X_{Q}$, and $P$ is left primitive if and only if $Q$ is left primitive.

Proof. (i) Let $P$ be a prime ideal of $S$. Choose $Q \in \mathscr{V}_{P}$. Note from [12, 5.3, 5.4] that $\mathrm{d}(R / Q)=\mathrm{d}\left(S / J_{Q}\right)$. From (3.2) we see that $P$ is the left annihilator of a factor $M$ of $S / T_{Q}$ such that ann $M_{R}=Q$. Hence $\mathrm{d}(R / Q)=\mathrm{d}(S / P)$. It now follows from $[12,3.16]$ that $P$ is minimal over $J_{Q}$. (Furthermore, since $\mathrm{d}(R / P \cap R)=\mathrm{d}(S / P)=\mathrm{d}(R / Q)$, by $[12,5.5]$, we see that $Q$ is minimal over $P \cap R$.) Part (i) follows.

(ii) First, it follows from [7, 7.16(b)] that if $Q$ is an arbitrary left primitive ideal of $R$ then each prime ideal in $X_{Q}$ is left primitive. Now suppose that $P$ 
is left primitive. Choose $Q \in \mathscr{V}_{P}$. The proof of (i) shows that $Q$ is minimal over $P \cap R$ and that $P \in X_{Q}$. From [7, 7.17] it follows that $Q$ is left primitive. Part (ii) follows.

(iii) Let $P$ be a prime ideal of $S$, and let $Q$ be an annihilator prime of ${ }_{R}(S / P)$. So there exists an $R-S$-sub-bimodule $B$ of $S / P$ with left annihilator equal to $Q$ and right annihilator equal to $P$. Thus [12, 5.3, 5.4] guarantees that $\mathrm{d}(S / P)=\mathrm{d}(R / Q)$. But $\mathrm{d}(S / P)=\mathrm{d}(R / P \cap R)$ by $[12,5.5]$. Hence $Q$ is minimal over $P \cap R$ by [12,3.16]. It follows from [7, 7.17] that $Q$ is left primitive if and only if $P$ is left primitive.

Now let $F=\operatorname{Fract}(S / P)$. Since $F Q B=0$, it follows that $F / F Q$ is a nonzero $F$-R-bimodule factor of $F$. Let $L$ denote a simple $F$-R-bimodule factor of $F / F Q$, and let $M$ denote the canonical $S$-R-bimodule image of $S$ in $L$. So ann $M=P$ and ann $M_{R}=Q^{\prime}$ where $Q^{\prime}$ is a prime ideal of $R$ containing $Q$. But then $\mathrm{d}(S / P)=\mathrm{d}\left(R / Q^{\prime}\right)=\mathrm{d}(R / Q)$ by the above paragraph and [12, 5.3, 5.4]. Hence $Q=Q^{\prime}$ by $[12,3.16]$. Now note that $M$ is torsionfree as a right $R / Q$-module by $[7,7.19(\mathrm{a})]$. Hence $P$ is the annihilator of the left $S$-module $M \otimes_{R} \operatorname{Fract}(R / Q)$. Since $M$ is an $S$-R-bimodule factor of $S$, we see that $P \supseteq J_{Q}$. But $\mathrm{d}(S / P)=\mathrm{d}(R / Q)=\mathrm{d}\left(S / J_{Q}\right)$, by $[12,5.3,5.4]$, and so $P$ is minimal over $J_{Q}$ by $[12,3.16]$. Part (iii) follows.

Remark 4.3. The conclusions of (4.2) remain true if instead of being algebras of finite GK-dimension, $R$ and $S$ are rings with a symmetric dimension function, as defined for example in [3]. In particular, the conclusions hold true if instead of the GK-dimension hypothesis it is assumed that $R$ and $S$ are rings satisfying the second layer condition, since classical Krull dimension is symmetric for such rings. See $[7,12.5]$ or $[10,8.2 .8]$.

\section{ACKNOWLEDGMENT}

The author is grateful to T. H. Lenagan for pointing out an error in a preliminary version of this note.

\section{REFERENCES}

1. M. Artin and W. Schelter, Integral ring homomorphisms, Adv. Math. 39 (1981), 289-329.

2. W. Borho, Invariant dimension and restricted extension of noetherian rings, Séminaire d'Algébre Paul Dubreil et Marie-Paule Malliavin, Lecture Notes in Math., vol. 924, Springer, New York, 1982, pp. 51-71.

3. __ On the Joseph-Small additivity principle for Goldie ranks, Compositio Math. 57 (1982), 3-29.

4. K. A. Brown, Localisation at cliques in group rings, J. Pure Appl. Algebra 41 (1986), 9-16.

5. K. A. Brown and R. B. Warfield, Jr., The influence of ideal structure on representation theory, J. Algebra 116 (1988), 294-315.

6. J. Dixmier, Idéaux primitifs dans l'algèbre enveloppante d'une algèbre de Lie semi-simple complexe, C. R. Acad. Sci. Paris Sér. A 272 (1971), 1628-1630.

7. K. A. Goodearl and R. B. Warfield, Jr., An introduction to noncommutative noetherian rings, London Math. Soc. Stud. Texts, vol. 16, Cambridge Univ. Press, Cambridge and New York, 1989.

8. T. J. Hodges and J. Osterburg, A rank two indecomposable projective module over a noetherian domain of Krull dimension one, J. London Math. Soc. (2) 19 (1987), 139-144. 
9. J. C. Jantzen, Einhullende algebren halbeinfacher Lie-algebren, Ergeb. Math. Grenzgeb. (3), vol. 3, Springer, New York, 1983.

10. A. V. Jategaonkar, Localization in Noetherian rings, London Math. Soc. Lecture Note Ser., vol. 98, Cambridge Univ. Press, Cambridge and New York, 1986.

11. A. Joseph and L. W. Small, An additivity principle for Goldie rank, Israel J. Math. 31 (1978), 105-114.

12. G. R. Krause and T. H. Lenagan, Growth of algebras and Gelfand-Kirillov dimension, Pitman, London, 1985.

13. T. H. Lenagan and R. B. Warfield, Jr., Affiliated series and extensions of modules, J. Algebra 142 (1991), 164-187.

14. E. S. Letzter, Prime ideals in finite extensions of noetherian rings, J. Algebra 135 (1990), 412-439.

15. __ Primitive ideals in finite extensions of noetherian rings, J. London Math. Soc. (2) 39 (1989), 427-435.

16. __ Prime and primitive ideals in enveloping algebras of solvable Lie superalgebras, Abelian Groups and Noncommutative Rings: A Collection of Papers in Memory of Robert B. Warfield, Jr. (L. Fuchs et al., eds.), Contemp. Math., Amer. Math. Soc., Providence, RI (to appear).

17. I. M. Musson, A classification of primitive ideals in the enveloping algebra of a classical simple Lie superalgebra Adv. Math. 91 (1992), 252-268.

18. D. S. Passman, Infinite crossed products, Academic Press, New York, 1989.

19. J. C. Robson, Prime ideals in intermediate extensions, Proc. London Math. Soc. (3) 44 (1982), 372-384.

20. L. H. Rowen, Polynomial identities in ring theory, Academic Press, New York, 1980.

21. J. C. McConnell and J. C. Robson, Noncommutative notherian rings, Wiley-Interscience, New York, 1988.

22. M. Scheunert, The theory of Lie superalgebras, Lecture Notes in Math., vol. 716, Springer, Berlin, 1979.

23. L. W. Small and R. B. Warfield, Jr., Finite extensions of rings. II, preprint, University of California, San Diego, 1988.

24. J. T. Stafford, The Goldie rank of a module, Noetherian Rings and Their Applications (L. W. Small, ed.), Math. Surveys Monographs, vol. 24, Amer. Math. Soc., Providence, RI, 1987.

25. D. Vogan, The orbit method and primitive ideals for semisimple Lie algebras, Lie Algebras and Related Topics, CMS Conf. Proc., vol. 5, Amer. Math. Soc., Providence, RI, 1986, pp. 281-316.

26. R. B. Warfield, Jr., Prime ideals in ring extensions, J. London Math. Soc. (2) 28 (1983), 453-460.

Department of Mathematics, University of Utah, Salt lake City, Utah 84112 